\title{
Consortia-mediated bioprocessing of cellulose to ethanol with a symbiotic Clostridium phytofermentans/yeast co-culture
}

\author{
Trevor R Zuroff', Salvador Barri Xiques ${ }^{2}$ and Wayne R Curtis $^{1^{*}}$
}

\begin{abstract}
Background: Lignocellulosic ethanol is a viable alternative to petroleum-based fuels with the added benefit of potentially lower greenhouse gas emissions. Consolidated bioprocessing (simultaneous enzyme production, hydrolysis and fermentation; (BP) is thought to be a low-cost processing scheme for lignocellulosic ethanol production. However, no single organism has been developed which is capable of high productivity, yield and titer ethanol production directly from lignocellulose. Consortia of cellulolytic and ethanologenic organisms could be an attractive alternate to the typical single organism approaches but implementation of consortia has a number of challenges (e.g., control, stability, productivity).

Results: Ethanol is produced from a-cellulose using a consortium of $C$. phytofermentans and yeast that is maintained by controlled oxygen transport. Both Saccharomyces cerevisiae cdt-1 and Candida molischiana "protect" C. phytofermentans from introduced oxygen in return for soluble sugars released by C. phytofermentans hydrolysis. Only co-cultures were able to degrade filter paper when mono- and co-cultures were incubated at $30^{\circ} \mathrm{C}$ under semi-aerobic conditions. Using controlled oxygen delivery by diffusion through neoprene tubing at a calculated rate of approximately $8 \mu \mathrm{mol} / \mathrm{L}$ hour, we demonstrate establishment of the symbiotic relationship between C. phytofermentans and S. cerevisiae cdt-1 and maintenance of populations of $10^{5}$ to $10^{6} \mathrm{CFU} / \mathrm{mL}$ for 50 days. Comparable symbiotic population dynamics were observed in scaled up $500 \mathrm{~mL}$ bioreactors as those in $50 \mathrm{~mL}$ shake cultures. The conversion of a-cellulose to ethanol was shown to improve with additional cellulase indicating a limitation in hydrolysis rate. A co-culture of C. phytofermentans and S. cerevisiae cdt- 1 with added endoglucanase produced approximately $22 \mathrm{~g} / \mathrm{L}$ ethanol from $100 \mathrm{~g} / \mathrm{L}$ a-cellulose compared to C. phytofermentans and S. cerevisiae cdt-1 mono-cultures which produced approximately 6 and $9 \mathrm{~g} / \mathrm{L}$, respectively.

Conclusion: This work represents a significant step toward developing consortia-based bioprocessing systems for lignocellulosic biofuels production which utilize scalable, environmentally-mediated symbiosis mechanisms to provide consortium stability.
\end{abstract}

Keywords: Consortia, Consolidated bioprocessing, Cellulosic ethanol, Symbiosis, Oxygen transport

\section{Background}

In nature microbes rarely live in isolation, but rather exist in highly diverse and complex communities referred to as consortia [1]. These consortia are often capable of tasks that are far too complex for any single organism to complete themselves including some of the most important global biogeochemical cycles [2]. The organisms

\footnotetext{
* Correspondence: wrc2@psu.edu

${ }^{1}$ Current address: The Pennsylvania State University, 158 Fenske Laboratory, University Park, PA 16802 USA

Full list of author information is available at the end of the article
}

living in these communities interact in numerous ways ranging from cooperation to direct competition [3]. Microbiologists and engineers have come to appreciate the diversity and capacity of natural microbial communities and large efforts have been undertaken to understand natural consortia and to engineer synthetic consortia for biotechnological purposes [4-7].

Two of the key challenges in society today are to reduce energy dependence on petroleum and reduce greenhouse gas emissions [8]. The transportation sector is a prime candidate for addressing these two challenges because it relies

\section{Biomed Central}


on petroleum for approximately $93 \%$ of its energy and releases almost as much carbon dioxide as both the commercial and residential sectors combined [9]. Lignocellulosic biomass has become an increasingly feasible source of carbohydrates for biological production of alternative fuels such as ethanol [10]. Consolidated bioprocessing (the simultaneous biological hydrolysis and fermentation of biomass; $\mathrm{CBP}$ ) is thought to be one of the most cost effective means of producing ethanol from lignocellulose $[11,12]$. However, no single organism has been isolated or genetically engineered to reach high enough ethanol concentrations, yields and productivities from lignocellulose [13]. Natural microbial consortia, on the other hand, are innately capable of high conversion of lignocellulosic biomass [see Table one in reference 7] but the resultant products (e.g., organic acids, $\mathrm{CO}_{2}, \mathrm{CH}_{4}$ ) are not suitable large-scale liquid transportation fuels.

An elegant example of a naturally occurring, lignocellulose degrading microbial consortium is the symbionts of the termite hindgut. Complex macromolecules are deconstructed in a series of steps that are facilitated by specific microbial species [14]. Protists produce cellulases for cellulose hydrolysis, while nitrogen fixing bacteria (e.g., Clostridia) sequester atmospheric nitrogen to compensate for the low nitrogen content of wood. Fermentative organisms consume soluble sugars released from cellulose hydrolysis to produce organic acids, $\mathrm{CO}_{2}$ and $\mathrm{H}_{2}$ which are subsequently converted by methanogens to methane [15]. This, along with other examples of natural microbial communities, has prompted interest in utilization of microbial consortia for lignocellulosic biofuels production $[5,7]$. However, unlike these natural systems, scientists are currently limited in our ability to generate stable, productive microbial communities. In order to successfully implement large scale consolidated bioprocessing of lignocellulosic materials for fuel ethanol production we must develop stable microbial consortia with the necessary functionality, process control and efficiency.

To transition from natural consortia which contain potentially hundreds of organisms to synthetic consortia containing several defined species, one must develop a mechanism for population control. Generating stable, controllable consortia has been a focus of recent work to genetically engineer mechanisms for establishing intraspecies consortia [16-19]. These approaches range from growth-controlling genetic circuits based on quorum sensing compounds [19] to complimentary auxotrophic amino acid exchange [17]. As proof-of-concept, these studies are quite elegant and encouraging but they may suffer on the large scale due to unstable and difficult genetic modifications in industrially relevant organisms. Others have demonstrated syntrophic interactions in a co-culture of Actinotalea fermentans with an engineered S. cerevisiae which produces methyl halides directly from cellulose
[20]. S. cerevisiae relieves acetate inhibition by converting it to methyl halides, which are precursors for various fuel compounds. However, synthetic syntrophic interactions may be unstable since at least one organism does not necessarily rely on the other for survival. Without an additional level of control (e.g., spatial structure [21-23]) the community may breakdown. Obligate mutualisms such as those described by You et al. 2004 and Shou et al. 2007 may be a more stable approach for consortia-mediated lignocellulosic ethanol production. With this in mind, symbiotic consortia of a wide range of organisms could be generated using various mechanisms based on both genetic and environmental control factors [7].

In this work we develop a symbiotic co-culture of the cellulolytic mesophile, Clostridium phytofermentans [24] and a cellodextrin fermenting yeast, Candida molischiana [25] or S. cerevisiae cdt-1 [26]. We establish a symbiosis (obligate mutualism) between $C$. phytofermentans and the yeast species by controlling the volumetric transport rate of oxygen. Both yeasts are capable of providing respiratory protection to the obligate anaerobe, C. phytofermentans, in return for soluble carbohydrates released from cellulose hydrolysis. The yeast converts these soluble carbohydrates to ethanol. At high substrate loading we noted a decreased conversion of cellulose by $C$. phytofermentans therefore endoglucanase was added to further evaluate the potential for improvements in the co-culture approach. In this cellulase-assisted format, the co-culture produces over twice as much ethanol and degrades two and three times as much $\alpha$-cellulose as the $S$. cerevisiae cdt-1 and C. phytofermentans monocultures, respectively. This work represents a significant step in utilizing a scalable environmental control mechanism (i.e. oxygen transport) to induce a stable symbiosis in a consortium of two diverse organisms. This general approach to symbiosis development is applicable to a variety of organisms, substrates and products and can be used to explore diverse consortia-mediated bioprocesses.

\section{Results and discussion}

\section{Design and development of C. phytofermentans/yeast symbiosis}

Oxygen introduction was proposed as a mechanism for establishing a symbiosis between mixtures of organisms in which one organism "protects" the other from oxygen inhibition in return for soluble carbohydrates released from cellulose [7]. In this work we investigated this mechanism and the subsequent performance of a combination of the cellulolytic obligate anaerobic mesophile, C. phytofermentans, with one of two cellodextrin fermenting yeasts, C. molischiana or S. cerevisiae cdt-1. C. phytofermentans was chosen for its ability to hydrolyze and ferment pure cellulose and natural lignocellulosic materials. For example, under consolidated bioprocessing (CBP) conditions (i.e., simultaneous biological hydrolysis 
A.

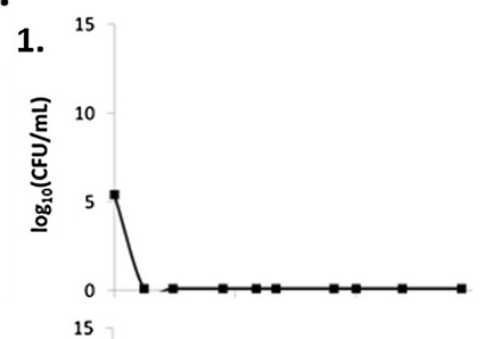

2.
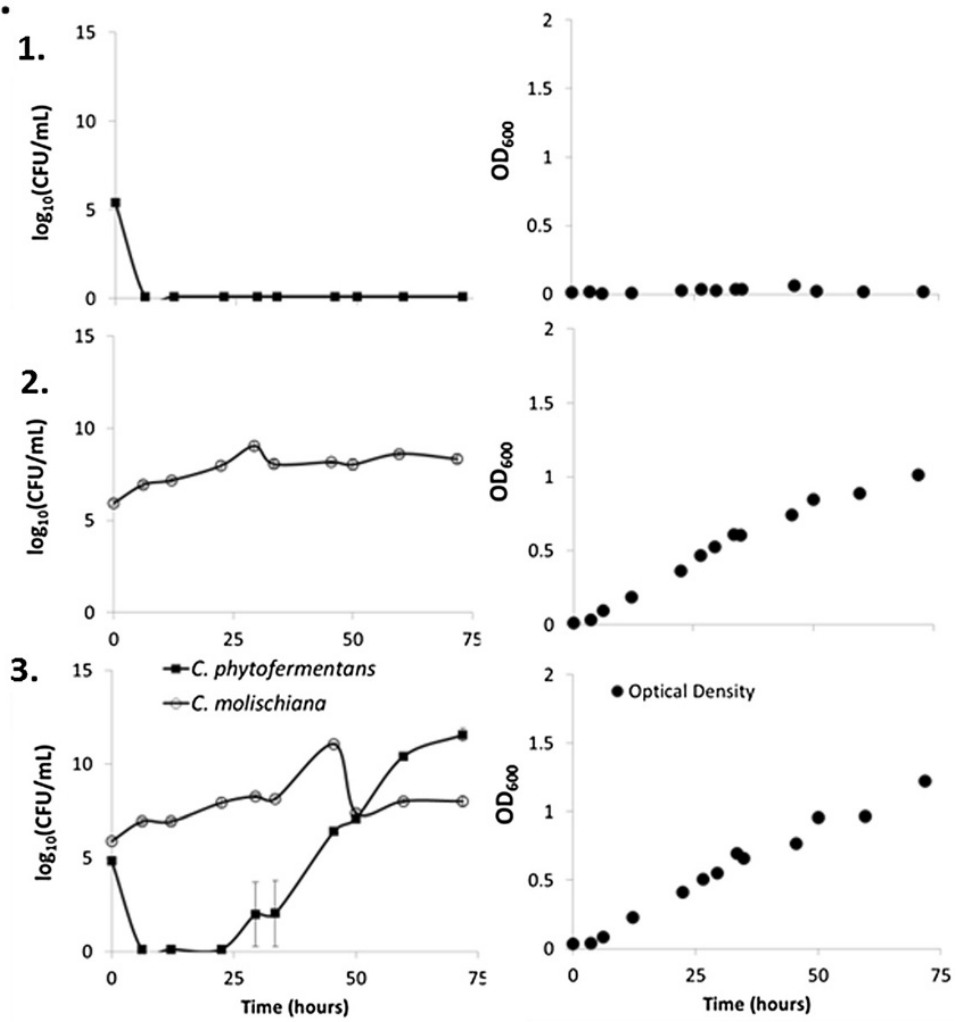

B.

1.
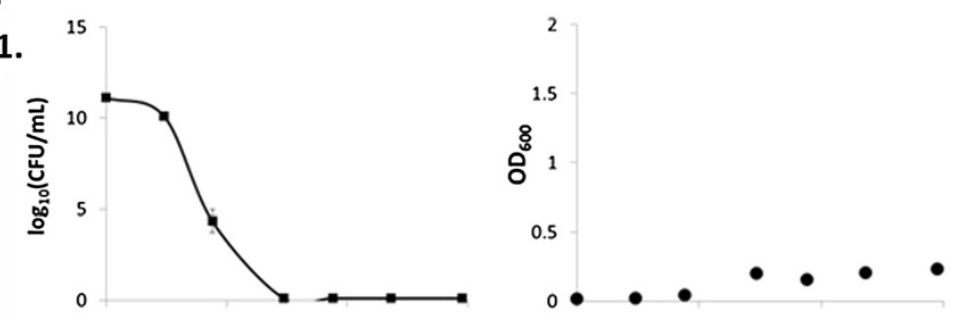

2. 15

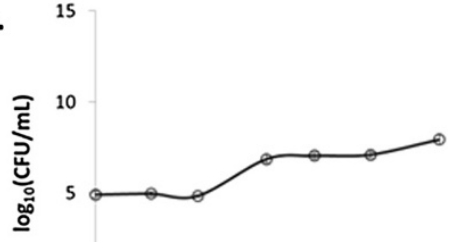

3.
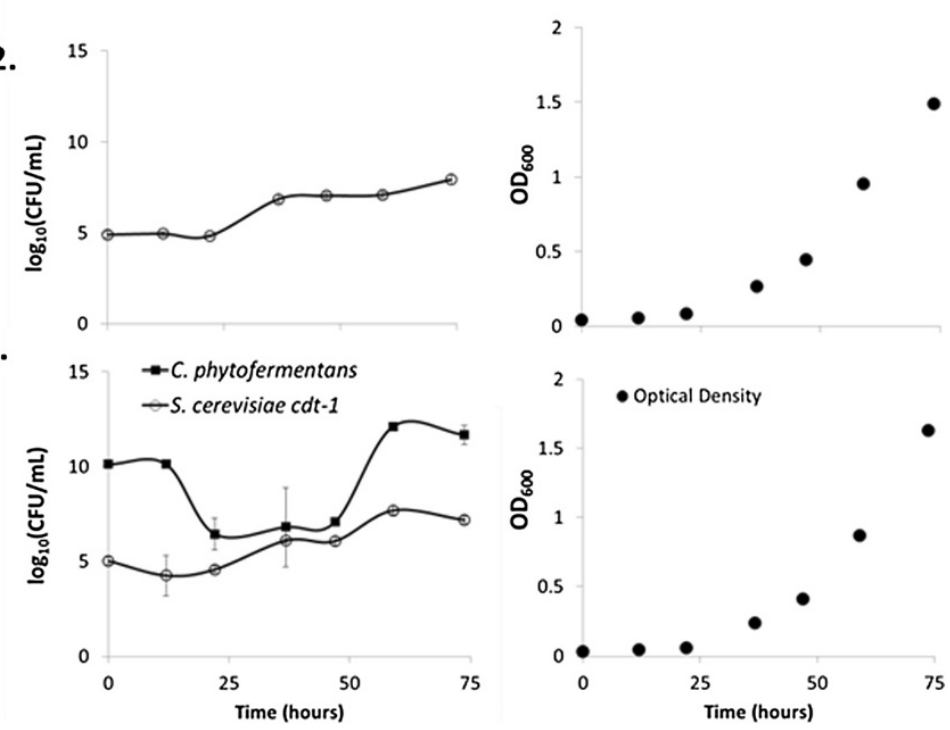
(See figure on previous page.)

Figure 1 Semi-aerobic mono- and co-culture growth on cellobiose. C. phytofermentans mono-culture (A.1 and B.1), C. molischiana mono-culture (A.2),C. phytofermentans/C. molischiana co-culture (A.3), S. cerevisiae cdt-1 mono-culture (B.2) and C. phytofermentans/S. cerevisiae cdt-1 co-Culture (B.3) viable cell counts (left) and optical densities (right) in GS2 cellobiose fermentations. Results are representative of at least two independent experiments. Error bars are the standard deviation among drops for colony counting.

and fermentation), C. phytofermentans was shown to hydrolyze $76 \%$ of glucan and $88.6 \%$ of xylan in AFEX treated corn stover and ferment it to $2.8 \mathrm{~g} / \mathrm{L}$ ethanol in 10 days [27]. C. molischiana is naturally able to ferment cellodextrins with degree of polymerization 2 to 6 to ethanol with yields of approximately $0.40 \mathrm{~g}$ ethanol/g substrate in 44 hours [28]. S. cerevisiae cdt-1 was engineered to import and cleave cellodextrins for subsequent fermentation (yield of approximately $0.44 \mathrm{~g} / \mathrm{g}$ cellobiose in about 100 hours) through expression of cellodextrin transporters and $\beta$-glucosidase from Neurospora crassa [26]. Although similar, the yeasts do have some important differences. For example, S. cerevisiae cdt-1 transports and cleaves cellodextrins internally [26] while C. molischiana does so externally with extracytoplasmic $\beta$-glucosidase [29]. These yeasts were chosen for their unique ability to ferment cellodextrins (not only glucose) which was hypothesized to increase ethanol yield and relieve cellulase inhibition by the soluble sugar products. In addition, these two organisms provide a comparison across two genus and between engineered and naturally occurring strains. In this study we used $\alpha$-cellulose (Sigma) as a model cellulosic substrate to study hydrolysis and fermentation since it avoids the presence of additional carbon sources (e.g., hemicellulose).

To test oxygen consumption and the resultant protection, cultures with $12 \mathrm{~g} / \mathrm{L}$ cellobiose as a mutually accessible carbon source, were inoculated with $10^{5}$ to $10^{6} \mathrm{CFU} / \mathrm{mL}$ of $C$. molischiana or S. cerevisiae cdt-1 and $10^{5}$ to $10^{10} \mathrm{CFU} / \mathrm{mL}$ C. phytofermentans. Dissolved oxygen was not removed from the medium at the beginning of the experiment however the cap was kept tight throughout the experiment aside from sampling. C. phytofermentans mono-cultures were unable to grow and viable cells dropped below detection $(<100 \mathrm{CFU} / \mathrm{mL}$ ) almost immediately with low starting concentration (Figure 1A.1.) and after a short lag with higher starting concentration (Figure 1B.1). C. molischiana and $S$. cerevisiae cdt- 1 in both mono- and co-culture grew relatively rapidly, showing increases in both viable cells and optical density (Figure 1A.2,3 and B.2,3). The discrepancy between the apparent length of the growth phase in optical density for $C$. molischiana mono-cutlures and viable cells is thought to be due to the insensitivity of CFU measurements and possibly a partial loss of viable cells which still contribute to OD. During rapid growth the yeast presumably consumed all available oxygen which, in co-culture, allowed for growth of C. phytofermentans (Figure 1A.3 and 1B.3). This result could be expected since similar relief of oxygen inhibition has been demonstrated in fungal/bacterial and bacterial/bacterial interactions [30,31] and is generally observed in naturally occurring microbial consortia [32]. However, this is the first demonstration utilizing an anaerobic cellulolytic bacteria and cellodextrin fermenting yeast. These results confirm that yeast provide respiratory protection to the obligate anaerobe, $C$. phytofermentans as an important component of the symbiotic cooperation that would be required for growth on cellulose as the sole carbon source.

This microbial interaction was expanded to include the complimentary component of the proposed symbiosis by providing carbohydrate that is released from cellulose by C. phytofermentans. Mono- and co-cultures were inoculated into medium containing Whatman \#1 filter paper strips under semi-aerobic conditions (i.e., initially oxygenated with tubes that allowed gas transfer). After approximately $15-40$ days of static incubation at $30^{\circ} \mathrm{C}$, only co-cultures showed degradation of filter paper (Figure 2). Here C. molischiana and S. cerevisiae cdt-1 provide respiratory protection to $C$. phytofermentans in exchange for soluble substrate provided from cellulose via $C$. phytofermentans hydrolysis. However, subsequent experimentation at increased oxygen transfer rates showed that these soluble products were a combination cellodextrins, glucose and ethanol produced by $C$. phytofermentans (data not shown). Protective mechanisms similar to this have been observed in mixed bacterial cultures [33] which supports the use of oxygen as a robust regulatory mechanism and suggests that flexibility exists in the selection of the facultative partner organism.

\section{Low level oxygen transfer promotes symbiosis while maintaining ethanol production}

To prevent ethanol consumption while maintaining sufficient oxygen to promote the symbiosis requires the use of controlled low level oxygen introduction. Submerged neoprene tubing with continuous internal air flow was used to transfer oxygen in two different scale reactor systems, $50 \mathrm{~mL}$ and $500 \mathrm{~mL}$ (Figure 3). Using this design, altering the submerged tubing length provides a simple means of altering the oxygen transfer rate. Assuming a constant driving force from air and a zero oxygen concentration in the culture (as determined visually by colorless resazurin) the oxygen transfer rate (OTR) through the tubing was estimated to be approximately $0.04 \mu \mathrm{mol} \mathrm{O} / 2 \mathrm{~cm}$ hour. With $10 \mathrm{~cm}$ of submerged tubing in $50 \mathrm{~mL}$ of medium the OTR $=8 \mu \mathrm{mol} \mathrm{O} / \mathrm{L}$ hour which corresponds to a culture volumetric transport rate of approximately 

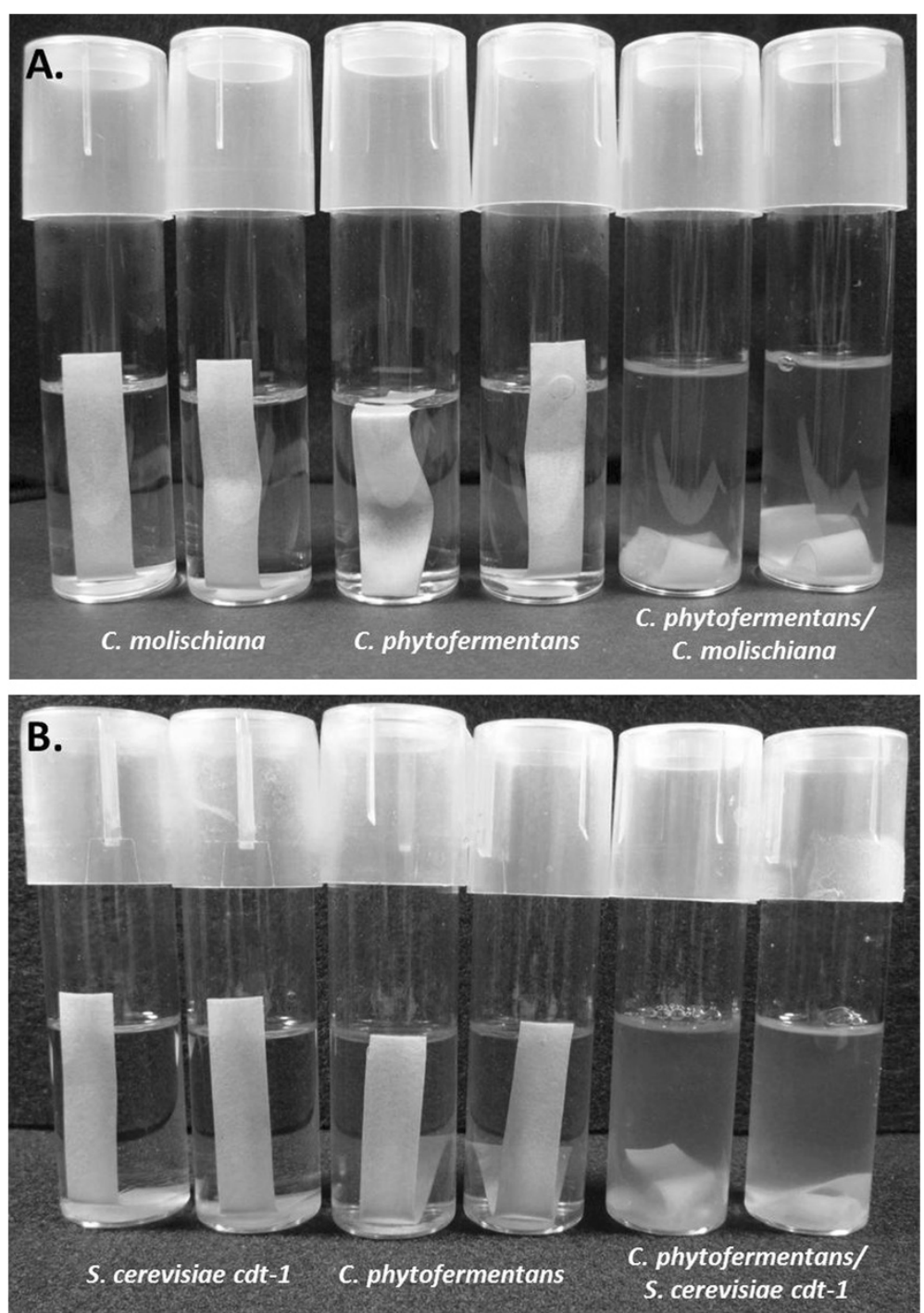

Figure 2 Static mono- and co-culture hydrolysis of filter paper. Semi-aerobic cultures in non-degassed GS2 medium with Whatman No. 1 filter paper strips as the carbon source. C. molischiana and C. phytofermentans mono- and co-cultures (A) and S. cerevisiae cdt-1 and C. phytofermentans mono- and co-cultures (B). Photographs taken after 40 days (A) and 15 days (B) static incubation at $30^{\circ} \mathrm{C}$ with periodic agitation. Image is representative of at least two independent experiments each with two replicates (as shown in the photographs).

0.03 hour $^{-1}$ (which is several orders of magnitude lower than typical shake flask fermentations [34]).

Using this OTR, C. molischiana and S. cerevisiae cdt-1 mono-cultures grew and fermented cellobiose with a lag phase of approximately 150-200 and 50-75 hours, respectively (Additional file $1 \mathrm{~B}$ and F). C. phytofermentans mono-cultures displayed only slightly inhibited growth and ethanol production compared to no oxygen controls (Additional file 1D). Without oxygen, growth and/or cellobiose consumption were not observed in C. molischiana mono-cultures while gradual cellobiose consumption was observed in S. cerevisiae cdt-1 mono-cultures (Additional file $1 \mathrm{~A}$ and $\mathrm{E}$ ). In fact, even with the addition of Ergosterol and Tween 80 (two common anaerobic growth factors for yeast [35]) and replacement of cysteine with glutathione [36] we were unable to achieve growth of C. molischiana on cellobiose under completely anaerobic conditions. $S$. cerevisiae cdt-1, on the other hand, displayed growth under these conditions (Additional file 2). This observation agrees well with other reports that indicated only a small number of yeast are able to grow in the complete absence of oxygen [37].

As expected, in anaerobic cellobiose co-culture $C$. molischiana and S. cerevisiae cdt-1 were outcompeted; the viable cell counts fell below detection $(<100 \mathrm{CFU} / \mathrm{mL})$ after about 100-200 hours (Additional file 3). On the other hand, co-cultures with oxygen transfer fermented all the cellobiose and supported the growth of both C. phytofermentans 

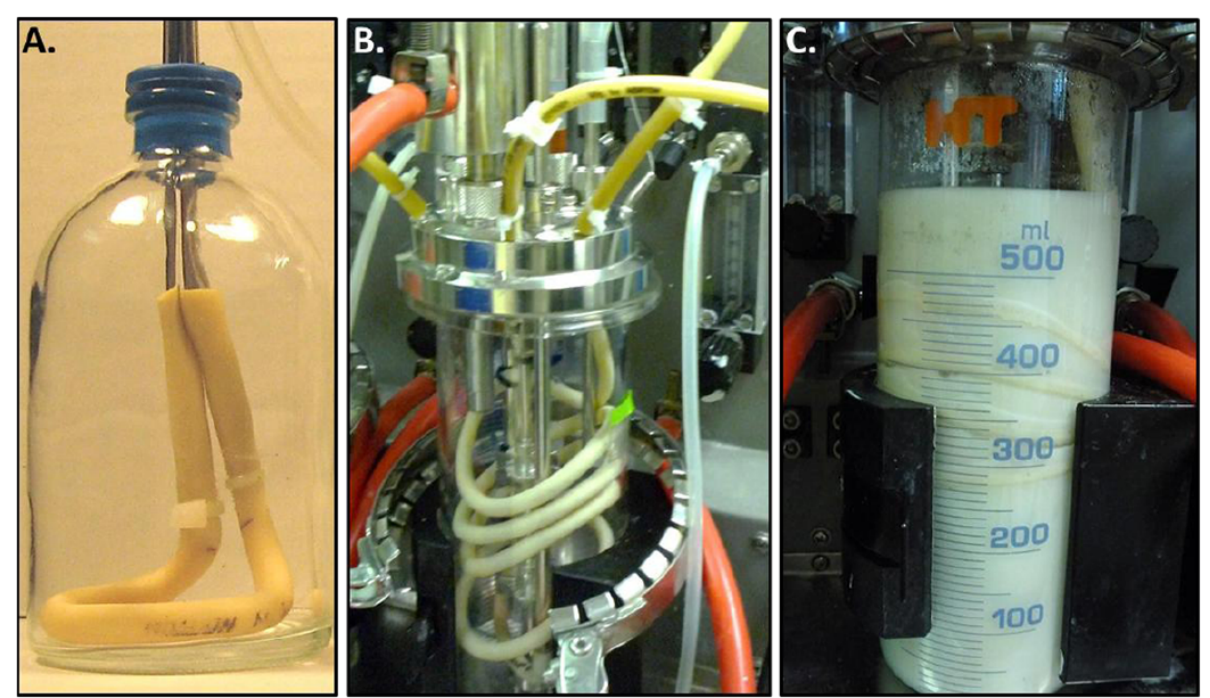

Figure 3 Bioreactors designed and utilized for diffusive oxygen transfer. Controlled oxygen transfer reactor with $10 \mathrm{~cm}$ neoprene tubing fixed to stainless steel tubing inserted into a sealed $100 \mathrm{~mL}$ serum bottle (A). Infors $500 \mathrm{~mL}$ reactor containing $100 \mathrm{~cm}$ neoprene tubing fixed to stainless steel outlet ports (B). Infors $500 \mathrm{~mL}$ reactor in operation with tubing loops submerged in the cellulose-containing medium (C).

and C. molischiana or S. cerevisiae cdt-1 (Additional file 3). The ethanol yield of the co-culture was slightly lower than that of the yeast mono-cultures since a small amount of acetate was produced early in the fermentation. In contrast to Figure 1, C. phytofermentans benefited early in the culture since the media was initially anaerobic while the increase in yeast viable cell counts occurred after approximately 100-200 hours during which time oxygen was continuously diffused into the culture (Additional file 3). This OTR appeared suitable for inducing and maintaining a coculture of $C$. phytofermentans and either C. molischiana or S. cerevisiae cdt-1 on cellobiose while allowing for high ethanol yield. Therefore, the same OTR was implemented in cellulose fermentations. In contrast to a recent coculture CBP study which required sequential culturing conditions for complimentary function while selectively inhibiting growth [38], this approach allows simultaneous growth and fermentation of both organisms which drastically simplifies culturing techniques.

\section{Stable co-culture cellulose fermentation}

Using low oxygen transfer rates as the symbiosis mediator, co-cultures were performed with $25 \mathrm{~g} / \mathrm{L} \alpha$-cellulose as the sole carbon source. S. cerevisiae cdt-1 was utilized in the remainder of the studies rather than $C$. molischiana due to its superior anaerobic growth and co-culture capabilities. C. phytofermentans dominated $\alpha$-cellulose cocultures during the first few hundred hours during which time yeast populations fell from about $10^{6}$ viable cells per $\mathrm{mL}$ to below detection (Figure $4 \mathrm{~A}$ and $\mathrm{B}$ ). Without oxygen, the yeast population remained at or below detectable limits in both co-culture (Figure 4A) and mono-culture
(Additional file 4A). In co-culture fermentations with added oxygen, the yeast population recovered to a maximum of about $10^{4}-10^{5} \mathrm{CFU} / \mathrm{mL}$ presumably due to simultaneous oxygen and carbon availability (Figure 4B). In fact, soluble cellodextrin concentrations in co-cultures with oxygen were consistently lower than those without oxygen even though cellulose hydrolysis was essentially identical (data not shown). As expected due to their inability to utilize cellulose, S. cerevisiae cdt-1 mono-cultures did not grow with or without added oxygen (Additional file 4A). C. phytofermentans growth was relatively unaffected by coculturing (Figure 4) and oxygen introduction (Additional file 4B). This confirms that oxygen can be used to generate a symbiotic relationship between $C$. phytofermentans which provides a soluble carbon source to $S$. cerevisiae cdt- 1 from cellulose in return for preventing oxygen inhibition. However, for these treatments no significant improvements in ethanol production or cellulose hydrolysis were observed in oxygen supplemented co-cultures relative to anaerobic C. phytofermentans mono-cultures (data not shown).

To investigate the scalability and attempt to improve performance of the co-culture, the fermentations were scaled up ten-fold (from $50 \mathrm{~mL}$ to $500 \mathrm{~mL}$ ) to an Infors Sixfors bioreactor system (Figure 3C) with enhanced mixing, $\mathrm{pH}$ control and an $\alpha$-cellulose concentration of $100 \mathrm{~g} / \mathrm{L}$. The OTR was maintained at approximately $0.4 \mu \mathrm{mol} \mathrm{O}_{2}$ /hour by increasing the submerged tubing length by a factor of ten from 10 to $100 \mathrm{~cm}$. Under these conditions, the population dynamics were essentially identical to the small scale reactors with only a slightly longer delay in S. cerevisiae cdt-1 re-growth (Figure 4C). Even with pH control, mixing and higher initial substrate concentration, the fermentation 

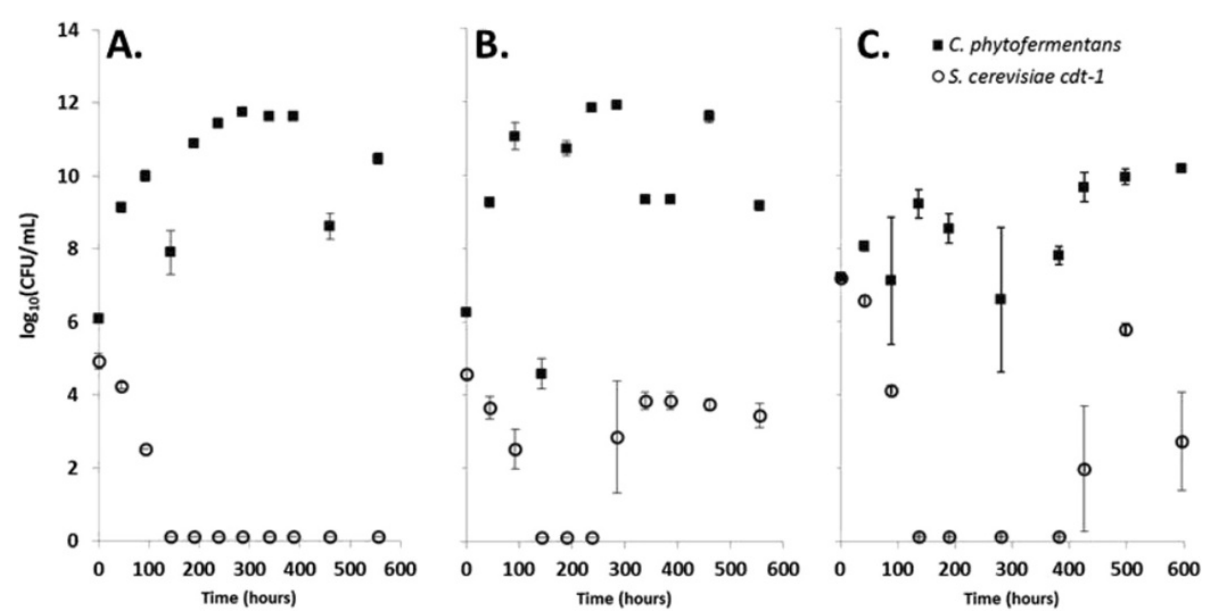

Figure 4 Co-culture population dynamics with diffusive oxygen transfer. Population dynamics for $50 \mathrm{~mL}, 25 \mathrm{~g} / \mathrm{L}$ a-cellulose co-culture fermentations without added oxygen (A) and with added oxygen (B) and $500 \mathrm{~mL}, 100 \mathrm{~g} / \mathrm{L}$ a-cellulose co-culture fermentation (C). Results in $\mathbf{A}$ and $\mathbf{B}$ are representative of at least 3 independent experiments. Results in $\mathbf{C}$ represent a single experiment. Error bars are plus and minus one standard deviation among the drops used for colony counting.

performance was not significantly improved compared to the small scale fermentations (data not shown). It is nonethe-less encouraging that the population control mechanism based on oxygen transport rates could be readily scaled. In addition to increasing tubing length it is easy to envision that the OTR could be maintained by altering tubing thickness, internal oxygen concentration or with minimal gas sparging at a larger scale.

The long term population stability of the co-culture was determined by growing C. phytofermentans and $S$. cerevisiae cdt-1 in co-culture with and without oxygen for 50 days. Serum bottles with 25 mL GS2 medium and $150 \mathrm{~g} / \mathrm{L} \alpha$-cellulose were inoculated with approximately equal cell concentration (about $10^{5} \mathrm{CFU} / \mathrm{mL}$ ) of C. phytofermentans and S. cerevisiae cdt-1. At 30, 40 and 50 days the individual cell concentrations were determined (Additional file 5A and B). The co-culture with added oxygen maintained $C$. phytofermentans and $S$. cerevisiae cdt-1 concentrations of $10^{5}$ to $10^{7} \mathrm{CFU} / \mathrm{mL}$ at every time point whereas, without oxygen, the yeast population fell below detection prior to the 30 day sample. The presence of yeast in the oxygen positive co-culture was also apparent in that no glucose was accumulated compared to almost $3 \mathrm{~g} / \mathrm{L}$ glucose accumulation in the coculture without oxygen (Additional file $5 \mathrm{C}$ and $\mathrm{D}$ ).

These results demonstrate the ability to effectively generate a stable, symbiotic co-culture of $C$. phytofermentans and $S$. cerevisiae cdt-1 via diffusion of oxygen at a rate of approximately $8 \mu \mathrm{mol} \mathrm{O}_{2} / \mathrm{L}$ hour. The oxygen transfer mechanism was scalable from $50 \mathrm{~mL}$ to $500 \mathrm{~mL}$. However, the fermentations were still limited in their hydrolytic capacity meaning the co-culture would not significantly outperform C. phytofermentans mono-cultures.
Co-culture simultaneous saccharifcation and fermentation The lack of increased performance of the co-culture relative to $C$. phytofermentans mono-culture appears to be due to an inability of $C$. phytofermentans to hydrolyze high solids loading cellulose to provide soluble substrate for the yeast partner. C. phytofermentans was repeatedly unable to fully utilize insoluble substrate concentrations in excess of approximately $30 \mathrm{~g} / \mathrm{L}$ (unpublished observation). To explore the potential for improving the co-culture approach we sought to increase soluble sugar yield by fermenting $\alpha$ cellulose with and without added endoglucanase from Trichoderma viride (3.2.1.4). This specific approach was taken to simulate additional hydrolysis capacity of $C$. phytofermentans while acknowledging the differences that exist between fungal and Clostridial cellulases.

Mono- and co-cultures were grown in $100 \mathrm{~mL}$ serum bottles with $100 \mathrm{~g} / \mathrm{L} \alpha$-cellulose in $50 \mathrm{~mL}$ of ETGtGS2 medium. After about 400 hours, C. phytofermentans monocultures without oxygen hydrolyzed approximately $22 \%$ of the $\alpha$-cellulose. With the addition of $0.4 \mathrm{~g} / \mathrm{L}$ endoglucanase, or $3.8 \mathrm{IU} / \mathrm{mL}$, conversion increased to about $42 \%$ but the majority of the released reducing sugars simply accumulated leaving almost $23 \mathrm{~g} / \mathrm{L}$ reducing sugars in the final sample (Figure 5A). C. phytofermentans mono-cultures with and without added endoglucanase resulted in about $6 \mathrm{~g} / \mathrm{L}$ ethanol suggesting a limitation in metabolic capacity of C. phytofermentans (Figure 5A). With oxygen, approximately $35 \%$ of the $\alpha$-cellulose was hydrolyzed in $S$. cerevisiae cdt-1 mono-cultures (with enzyme) with about $1 \mathrm{~g} / \mathrm{L}$ reducing sugars accumulated and $9 \mathrm{~g} / \mathrm{L}$ ethanol produced. Co-cultures without added enzyme behaved essentially identical to C. phytofermentans mono-cultures consistent with hydrolysis-limited productivity. On the 


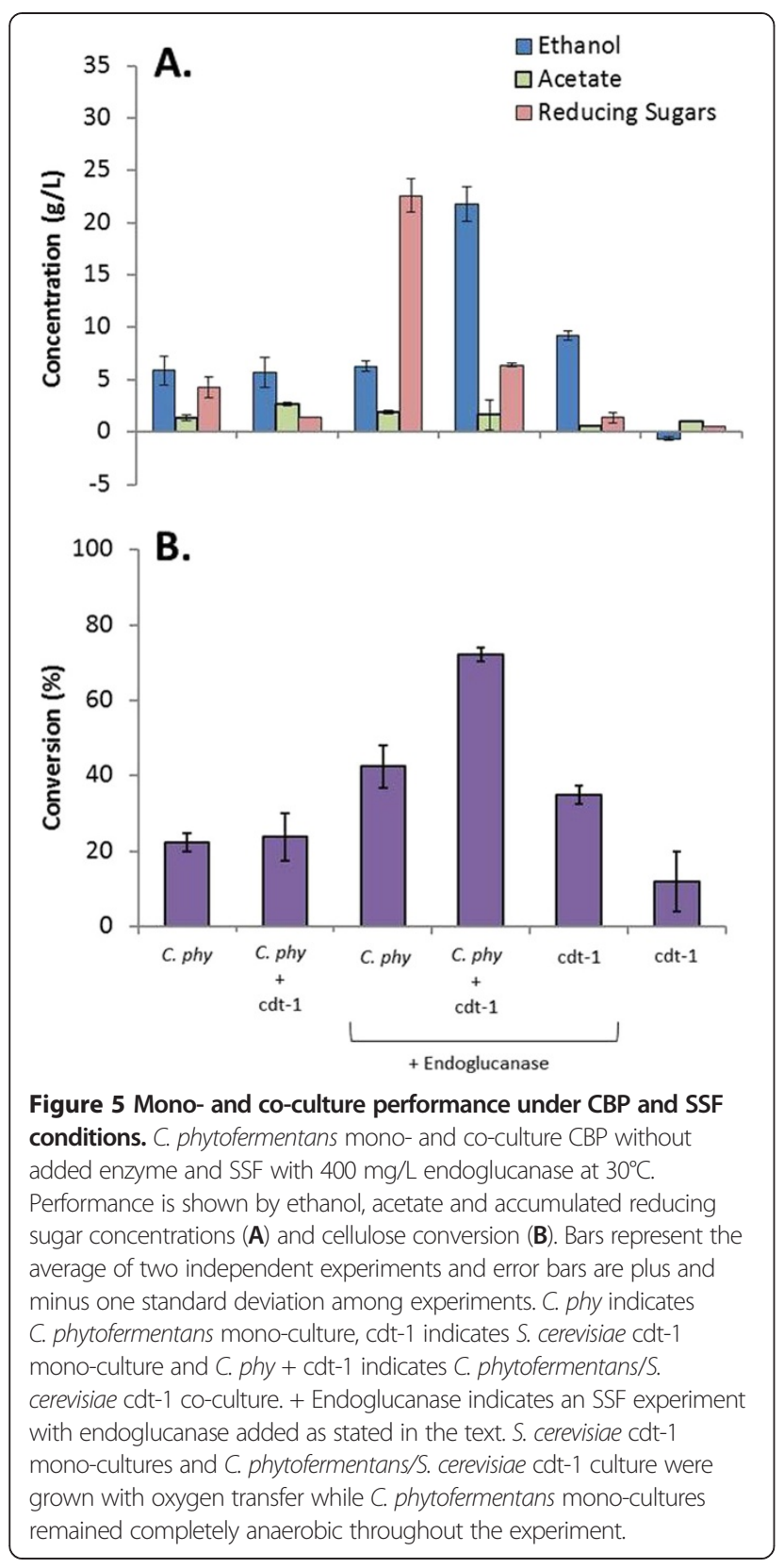

other hand, the co-culture with oxygen and endoglucanase produced more than two times more ethanol than either mono-culture with a final concentration of approximately $22 \mathrm{~g} / \mathrm{L}$ and hydrolyzed twice as much cellulose with a conversion of about $72 \%$ (Figure 5). A single experiment was incubated further and with an additional 240 hours the coculture reached almost $30 \mathrm{~g} / \mathrm{L}$ ethanol and $90 \%$ cellulose conversion (Additional file 6). The C. phytofermentans mono-culture with and without endoglucanse failed to produce additional ethanol and the $S$. cerevisiae cdt- 1 monocultures reached only $13 \mathrm{~g} / \mathrm{L}$ after the additional 240 hours of incubation (Additional file 6).
These results demonstrate that co-culture with $C$. phytofermentans significantly enhances simultaneous saccharification and fermentation of $\alpha$-cellulose with $S$. cerevisiae cdt-1. It is important to note in Figure 5 that the maximum productivity was observed for the consortium relative to the mono-culture even when exogenous cellulase was provided. This suggests there is considerable room for optimizing the productivity, and contrary to concerns of complexity, the symbiotic consortium is inherently stable. This synergistic interaction produced a final ethanol concentration that was $30 \%$ greater than the sum of the mono-culture titers $(22 \mathrm{~g} / \mathrm{L}$ versus $15 \mathrm{~g} / \mathrm{L})$. The improvements shown here are likely due to the combination of endoglucanse and the enzyme repertoire of C. phytofermentans relative to the single enzyme (endoglucanase) used in S. cerevisiae cdt-1 mono-cultures. The relief of feedback inhibition on T. viride endoglucanase by consumption of accumulated sugars and decreased $\mathrm{pH}$ caused by $C$. phytofermentans acetate production also likely contributed to the observed improvements. It is important, however, to note that the media conditions are optimized for co-culture growth and not necessarily mono-culture SSF productivity so changes in culturing conditions could lead to improved mono-culture performance. Independent of these subtle interpretations, these results unambiguously demonstrate the power of symbiotic microbial consortia in cellulosic ethanol production. The results also suggest that with improvements in $\mathrm{C}$. phytofermentans hydrolytic capacity, the co-culture has the potential to reach commercially relevant ethanol concentrations from $\alpha$-cellulose.

\section{Conclusions}

We have demonstrated the development, verification and application of a symbiotic co-culture of a cellulolytic mesophile, C. phytofermentans, and the cellodextrin fermenting yeast, C. molischiana or S. cerevisiae cdt-1 for direct ethanol production from $\alpha$-cellulose. Controlled oxygen transfer is used to induce a symbiosis between the two organisms in which the yeast removes oxygen, protecting C. phytofermentans, in return for soluble carbohydrates liberated from cellulose (Figure 6). The symbiotic co-cultures were stable for almost 2 months, hydrolyzed cellulose under semi-aerobic conditions and produced more ethanol from $\alpha$-cellulose via SSF than C. phytofermentans or S. cerevisiae cdt-1 mono-cultures. The addition of a moderate level of cellulase $400 \mathrm{mg} / \mathrm{L}$ (3.2.1.4 from T. viride) to the co-cultures in SSF experiments improved ethanol production two-fold greater than $S$. cerevisiae cdt-1 mono-culture and approximately fourfold greater than $C$. phytofermentans mono-cultures giving a final concentration of approximately $22 \mathrm{~g}$ ethanol/L after 400 hours. These results suggest that $C$. phytofermentans hydrolysis rates are well-matched to its natural metabolic 


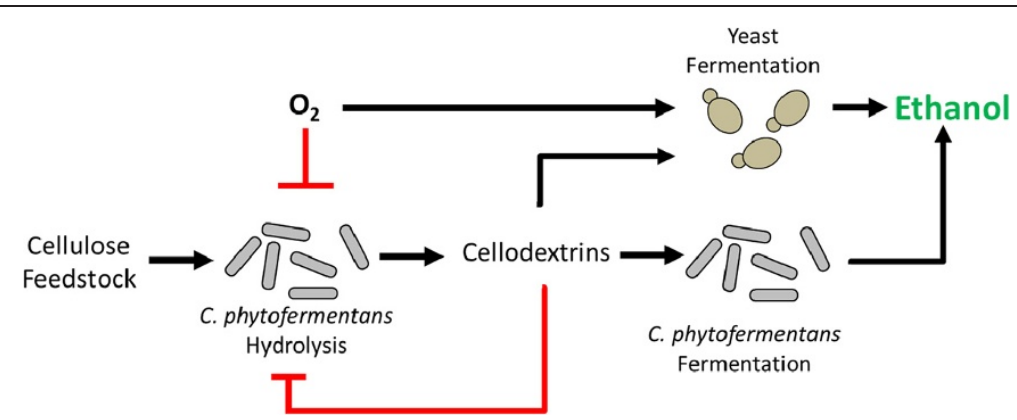

Figure 6 Proposed symbiotic consortium design. C. phytofermentans-yeast symbiotic consortium design based on cellulose hydrolysis to soluble cellodextrins which are utilized by yeast. Both C. phytofermentans and yeast produce ethanol from cellodextrins. Cellodextrins feedback inhibit cellulose hydrolysis, oxygen inhibits anaerobic bacterial growth while yeast oxygen consumption relieves inhibition.

rates and increased cellulase activity is needed to support a highly productive symbiosis. In addition, oxygen introduction as a mechanism for symbiosis establishment in microbial consortia was scaled from $50 \mathrm{~mL}$ to $500 \mathrm{~mL}$ and can be readily scaled up by conventional gas transfer at low cost making it highly applicable to industrial fermentation.

This work represents a significant advancement in establishing and controlling a microbial consortium of diverse organisms for biochemical production. In contrast to other approaches for generating symbiotic consortia that require genetic engineering, oxygen introduction is a simple, scalable technique that could be applied to organisms where genetic engineering tools are not yet available. However, both approaches have significant merit and genetic modification combined with an environmental control mechanism, like oxygen tension, could be an extremely powerful approach to consortia-mediated bioprocessing. The successful development of controllable, stable and productive engineered microbial consortia is likely to be a key advancement in many aspects of biotechnology. This approach combines complex functionality that is distributed across numerous organisms that can be independently optimized for a specific function. To gain better understanding, control and predictive capabilities, community mass and energy balance and metabolic models [39-41] may prove quite useful. Modeling combined with ecological theory could be applied to predict the fate of the populations in a consortium and the relative fitness and/or productivity of the individuals within the overall community.

Oxygen-facilitated symbiosis should be pursued as a mechanism for generating stable consortia of various other organisms including other cellulolytic bacteria and fungi as well as pentose and hexose fermenting yeasts and bacteria. In addition, other products, interactions and processes can be explored by incorporating other naturally occurring or genetically engineered organisms that have unique metabolic properties such as the production of biofuel molecules with improved energy density. As a model system, this symbiotic consortium can be used to explore the structural and functional aspects of cellulose degrading microbial communities. The work described herein used pure cellulose as a substrate but more complex lignocellulosic substrates must be investigated. The appropriate combination of organisms in a symbiotic consortium could result in an efficient consortia-mediated bioprocess which could improve the economic feasibility of lignocellulosic biofuels.

\section{Methods}

\section{Media and chemical sources}

GS2 medium [42] was used in all experimental cultures with the following composition per liter: $6 \mathrm{~g}$ Yeast Extract (BD), $2.9 \mathrm{~g} \mathrm{~K}_{2} \mathrm{HPO}_{4}$ (JT Baker), $2.1 \mathrm{~g}$ Urea (Sigma), $1.5 \mathrm{~g}$ $\mathrm{KH}_{2} \mathrm{PO}_{4}$ (JT Baker), 3 g Tri-Na Citrate $\mathrm{H}_{2} \mathrm{O}$ (Fischer), 2.33 g Cysteine $\mathrm{HCl} \mathrm{H} \mathrm{H}_{2} \mathrm{O}$ (MP Biomedical), 1 g MOPS (Sigma), 0.1 g $\mathrm{MgCl}_{2} \quad 6 \mathrm{H}_{2} \mathrm{O}$ (JT Baker), $0.0113 \mathrm{~g} \mathrm{CaCl}_{2}$ Annhydrous (Fischer), and 0.000125 g FeSO $_{4} \quad 7 \mathrm{H}_{2} \mathrm{O}$ (Fischer) and $0.01 \%$ Resazurin (Aldrich). The salts solution $\left(\mathrm{MgCl}_{2} 6 \mathrm{H}_{2} \mathrm{O}, \mathrm{CaCl}_{2}\right.$ Annhydrous and $\left.\mathrm{FeSO}_{4} 7 \mathrm{H}_{2} \mathrm{O}\right)$ and resazurin solution were made and sterilized separately and added after autoclave. The $\mathrm{pH}$ of the basal components was adjusted to 7 prior to sterilization. The concentration of $\alpha$-Cellulose (Sigma) was as described in the text. For inoculum cultures $\alpha$-Cellulose was replaced with $3 \mathrm{~g} / \mathrm{L}$ Cellobiose. The GS2 medium was modified for $\alpha$-cellulose co-cultures to contain $10 \mathrm{mg} / \mathrm{L}$ Ergosterol and $420 \mathrm{mg} / \mathrm{L}$ Tween 80 and $4.09 \mathrm{~g} / \mathrm{L}$ Glutathione in place of $2.33 \mathrm{~g} / \mathrm{L}$ Cysteine $\mathrm{HCl} \mathrm{H}_{2} \mathrm{O}$ (termed ETGtGS2). Either GS2 medium without cysteine and/or glutathione or YPC (5 g/L yeast extract, $10 \mathrm{~g} / \mathrm{L}$ peptone and $3 \mathrm{~g} / \mathrm{L}$ cellobiose) was used for cultivation of $C$. molischiana and $S$. cerevisiae cdt- 1 . Half strength Sabouraud medium contained (per liter) $5 \mathrm{~g}$ Casien Digest (Sigma), 5 g Dextrose (EM Sciences) and $10 \mathrm{~g}$ Agar (BD).

\section{Inoculation}

Initial cultures of Clostridium phytofermentans ISDgT were prepared from cryogenically $\left(-80^{\circ} \mathrm{C}\right)$ frozen stocks 
in $7.5 \mathrm{~mL}$ screw cap tubes with $5 \mathrm{~mL}$ GS2 medium. Oxygen was removed from C. phytofermentans culture medium by degassing (until de-pinked) in a Coy Anaerobic Chamber with a $1.5 \% \quad \mathrm{H}_{2} / 98.5 \% \quad \mathrm{~N}_{2}$ atmosphere. C. phytofermentans inoculum cultures were allowed to grow at $30^{\circ} \mathrm{C}$ to an $\mathrm{OD}_{600}$ of about 0.3 . Cultures were then restarted by placing $100 \mu \mathrm{L}$ of the initial culture into $4.9 \mathrm{~mL}$ of fresh GS2 medium. Experiments were inoculated by injecting approximately $2-3 \mathrm{~mL}$ of restarted cultures $\left(\mathrm{OD}_{600}\right.$ of about $\left.0.2-0.3\right)$ to give an initial $\mathrm{OD}_{600}$ of approximately $0.0125-0.025$. The amount of substrates and products in the inoculum was determined by HPLC and was subtracted from the final concentrations in the cultures to determine true yields.

For cellobiose co-culture experiments and filter paper degradation experiments yeast cultures were grown in GS2 medium without cysteine and/or glutathione under aerobic conditions at $30^{\circ} \mathrm{C}$ until reaching an $\mathrm{OD}_{600}$ of at least 0.3 then restarted. The cultures were then restarted and the restarted cultures were used as inoculum to obtain an initial $\mathrm{OD}_{600}$ of approximately $0.0125-0.025$. For the $\alpha$-cellulose experiments yeast cultures were grown in YPC medium at $30^{\circ} \mathrm{C}$ until reaching an $\mathrm{OD}_{600}$ of at least 0.5 , were restarted in YPC and allowed to grow until reaching an $\mathrm{OD}_{600}$ of approximately 1 and were then concentrated and used to inoculate with an initial $\mathrm{OD}_{600}$ of about 0.05 .

\section{Experimental cultures}

Fermentations were conducted in $100 \mathrm{~mL}$ serum vials sealed with butyl rubber stoppers and incubated at $30^{\circ} \mathrm{C}$ with $200 \mathrm{rpm}$ agitation or in the $500 \mathrm{~mL}$ Infors Sixfors bioreactor system at $30^{\circ} \mathrm{C}$ with $300 \mathrm{rpm}$ stirring. In the Sixfors bioreactor system, $\mathrm{pH}$ was controlled using addition of $1 \mathrm{~N}$ sodium hydroxide. Cultures were degassed in an anaerobic chamber to remove oxygen at the beginning of the experiment as described previously. A loop of neoprene tubing $(\mathrm{OR}=3.175 \mathrm{~mm}$, IR $=1.45 \mathrm{~mm}$ ) was submerged in the culture medium and attached at each end to stainless steel tubing inserted through the butyl stopper. In oxygen positive cultures, air was allowed to flow through the neoprene tubing at a flow rate of about $100 \mathrm{~L} / \mathrm{hr}$ using an aquarium pump. The stainless steel tubing was fashioned in a loop for oxygen negative cultures so that no oxygen could enter the culture.

Static hydrolysis experiments were conducted in $30 \mathrm{~mL}$ flat-bottom test tubes and incubated at $30^{\circ} \mathrm{C}$ with periodic agitation. Experimental media was not degassed prior to inoculation and residual carbon was not removed from the inoculum. Resazurin was not included in the media.

Simultaneous saccharification and fermentation experiments were conducted in $100 \mathrm{~mL}$ serum vials sealed with butyl rubber stoppers and incubated at $30^{\circ} \mathrm{C}$ with $200 \mathrm{rpm}$ agitation as previously described. Endoglucanase (3.2.1.4 from Trichoderma viride) was suspended in phosphate buffered saline, degassed and injected to reach a final concentration of $400 \mathrm{mg} / \mathrm{L}$. Cultures without endoglucanase received degassed, phosphate buffered saline only. One way valves attached to needles were inserted through the butyl stopper to release the gas produced via fermentation.

\section{Dry weight measurements}

Cellulose dry weight samples $(1 \mathrm{~mL})$ were removed from reactors with a syringe and placed into pre-tared $1.7 \mathrm{~mL}$ eppi-tubes (VWR). The samples were centrifuged at $14,000 \times \mathrm{g}$ for 10 minutes and the supernatant was removed (used as HPLC sample as described in the following section). The cellulose pellet was washed with DI $\mathrm{H}_{2} \mathrm{O}$ and again centrifuged at $14,000 \times \mathrm{g}$ for 10 minutes. The supernatant was removed and the tube was placed, open, in a drying oven at $70^{\circ} \mathrm{C}$. The samples were dried until reaching a constant mass and then weighed. The difference between the final and initial weight of the tube was assumed to be the dry mass of cellulose neglecting cellular mass.

\section{Fermentation product determination}

Cellobiose, glucose, ethanol and acetate concentrations were determined from supernatant (as described previously) taken from each culture and frozen immediately at $-20^{\circ} \mathrm{C}$. Following the completion of the experiment the samples were thawed and filtered into $1.5 \mathrm{~mL}$ screw cap vials (Agilent) using $0.45 \mu \mathrm{m}$ nylon syringe filters (Whatman). Samples were analyzed on an Agilent 1100 HPLC with a Jasco RI-1531 refractive index detector (RID) using an Aminex HPX-87H Cation exchange column (BioRad) with the appropriate guard column. Filtered (through 0.22 um filter) $0.01 \mathrm{M}$ sulfuric acid was used as the mobile phase, the column temperature was set at $65^{\circ} \mathrm{C}$ and the RID was set at $30^{\circ} \mathrm{C}$. Samples were injected at a volume of $25 \mathrm{uL}$ and the operating flow rate was $0.6 \mathrm{~mL} / \mathrm{min}$. Product formation reflects correction to the initial concentration in the medium immediately following inoculation. Therefore, carryover of low concentrations of fermentation products and subsequent consumption of these products (e.g., ethanol) may lead to negative production values.

\section{Soluble carbohydrate determination}

Soluble carbohydrates were determined using the anthrone-sulfuric acid colorimetric assay of Dreywood (Dreywood, 1946) adapted to a 96-well plate (Leyva et al. 2008). Breifly, $50 \mu \mathrm{L}$ of appropriately diluted sample was mixed with $150 \mu \mathrm{L}$ anthrone reagent $(2 \mathrm{~g} / \mathrm{L}$ anthrone in 98\% sulfuric acid) in a polypropylene 96-well plate and covered with a nylon adhesive cover. The 96-well plates were incubated at $4^{\circ} \mathrm{C}$ for 10 minutes, $100^{\circ} \mathrm{C}$ for $20 \mathrm{mi}$ nutes and room temperature for 20 minutes. The nylon 
cover was then removed and the plate was read in a Spectramax 384 Plus spectrophotometer at $620 \mathrm{~nm}$. The soluble carbohydrate concentration was determined using a known concentration standard curve that was run with each 96-well plate used.

\section{Colony forming unit determination}

C. phytofermentans and yeast populations were determined by performing serial dilutions of experimental samples in 96-well plates and subsequent drop plating of each dilution on the appropriate medium. C. phytofermentans was plated on GS2 medium with $6 \mathrm{~g} / \mathrm{L}$ lactose and was incubated at $30^{\circ} \mathrm{C}$ in an anaerobic chamber. C. molischiana and $S$. cerevisiae cdt- 1 were plated on $1 / 2$ Sabouraud medium and incubated at $30^{\circ} \mathrm{C}$ under aerobic conditions. Co-culture samples were plated on both types of plates and incubated under their respective conditions. Colonies were counted on dilutions that contained approximately 3 to 30 colonies per drop. Due to the non-soluble nature of cellulose which may act to trap, attach to or reject cells, colony counts often displayed significant variability but trends were found to be consistent across experiments.

\section{Additional files}

Additional file 1: Mono-culture growth and cellobiose fermentation performance with and without diffusive oxygen transfer.

Representative OD (top figures) and consumption/production profiles (bottom figures) for C. molischiana mono-cultures without oxygen (A), C. molischiana mono-cultures with oxygen (B), C. phytofermentans mono-cultures without oxygen (C),C. phytofermentans mono-cultures with oxygen (D), S. cerevisiae cdt-1 without oxygen (E) and S. cerevisiae cdt-1 with oxygen (F). Cellobiose (filled diamonds) and ethanol (open circles) are in grams per liter. Other products (e.g., glucose and acetate) are not shown for simplicity. Data is representative of at least two independent experiments.

Additional file 2: Growth of S. cerevisiae cdt-1 and C. molischiana in improved media under anaerobic conditions. Growth of $S$. cerevisiae cdt-1 (squares) and C. molischiana (circles) in GS2 medium containing ergosterol, Tween 80 and glutathione in place of cysteine. Closed and open symbols represent two different experimental trials where each symbol is the average of two replicates. Error bars denote plus and minus one standard deviation between the replicates. Note that aerobic controls of both organisms grew well under these conditions.

Additional file 3: Co-culture growth and cellobiose fermentation performance with and without diffusive oxygen transfer. Representative CFU/mL (top figures) and consumption/production profiles (bottom figures) for C. phytofermentans/C. molischiana co-cultures (A) and C. phytofermentans/S. cerevisiae cdt-1 co-cultures (B) Cellobiose (filled diamonds) and ethanol (open circles) are in grams per liter. Data is representative of at least two independent experiments.

Additional file 4: Mono-culture population dynamics with and without diffusive oxygen transfer. Population dynamics for $50 \mathrm{~mL}$, $25 \mathrm{~g} / \mathrm{L}$ a-cellulose $\mathrm{S}$. cerevisiae cdt-1 mono-culture fermentations (A) and C. phytofermentans mono-culture fermentations (B). Results are representative of at least 3 independent experiments and error bars are plus and minus one standard deviation among the drops used for colony counting.

Additional file 5: Co-culture populations and soluble sugar accumulation when grown on a-cellulose with and without oxygen. Viable cell counts at 30, 40 and 50 days for C. phytofermentans/S. cerevisiae cdt-1 co-cultures without oxygen (A) and with oxygen (B).
Accumulated soluble sugars (including glucose) and glucose concentration at 30, 40 and 50 days for C. phytofermentans/S. cerevisiae cdt-1 co-cultures without oxygen (C) and with oxygen (D). Bars are the average of two replicates and error bars represent plus and minus one standard deviation.

Additional file 6: Mono- and co-culture performance under CBP and SSF conditions after 640 hours. C. phytofermentans mono- and co-culture CBP without added enzyme and SSF with $400 \mathrm{mg} / \mathrm{L}$ endoglucanase at $30^{\circ} \mathrm{C}$. Performance is shown by ethanol and acetate concentrations (A) and cellulose conversion (B). Bars represent the average of two replicates in a single experiment and error bars are plus and minus one standard deviation among replicates. C. phy indicates C. phytofermentans mono-culture, cdt-1 indicates $S$. cerevisiae cdt-1 mono-culture and C. phy $+\mathrm{cdt}-1$ indicates C. phytofermentans/S. cerevisiae cdt-1 co-culture. + Endoglucanase indicates an SSF experiment with endoglucanase added as stated in the text. S. cerevisiae cdt-1 mono-cultures and C. phytofermentans/S. cerevisiae cdt-1 culture were grown with oxygen transfer while C. phytofermentans mono-cultures remained completely anaerobic throughout the experiment.

\section{Abbreviations}

AFEX: Ammonia fiber expansion; $\mathrm{OD}_{600}$ : Optical density at $600 \mathrm{~nm}$; CBP: Consolidated bioprocessing; OTR: Oxygen transfer rate; HPLC: High performance liquid chromatography; CFU: Colony forming unit; IU: International units; SSF: Simultaneous saccharificaiton and fermentation.

\section{Competing interests}

The authors declare that they have no competing interests.

\section{Author contributions}

TZ and WC conceived and designed the study. TZ carried out the majority of the experiments and drafted the manuscript. SX conducted the $C$. phytofermentans/C. molischiana static hydrolysis experiments and analysis as well as participated in media design. TZ and WC edited the draft. All authors read and approved the final manuscript.

\section{Acknowledgements}

We are grateful to Dr. Jamie Cate at the University of California, Berkeley for providing Saccharomyces cereivisae strain cdt-1, Dr. Susan Leschine at the University of Massachusetts, Amherst for Clostridium phytofermentans ISDg10 and the USDA NRRL for Candida molischiana (Y-2237). We thank Patrick Hillery, Jessica Bigham and Taylor Maher who assisted in sample collection and analysis and Mark Signs who supported analytical work. We would like to thank Thomas K. Wood at The Pennsylvania State University for his comments on the research and manuscript. This material is based upon work done by TR Zuroff supported by the National Science Foundation Graduate Research Fellowship under Grant No. DGE-0750756. TR Zuroff also received funding for this work from the Pennsylvania State University Department of Chemical Engineering John and Jeanette McWhirter Graduate Research Fellowship. Any opinions, findings, and conclusions or recommendations expressed in this material are those of the author(s) and do not necessarily reflect the views of the National Science Foundation.

\section{Author details}

${ }^{1}$ Current address: The Pennsylvania State University, 158 Fenske Laboratory, University Park, PA 16802 USA. ${ }^{2}$ Industrial Engineering Department, ETS IQS, Via Augusta 390, 08017 Barcelona, Spain.

\section{Received: 19 October 2012 Accepted: 18 April 2013}

Published: 29 April 2013

\section{References}

1. Davey ME, O'toole GA: Microbial biofilms: from ecology to molecular genetics. Microbiol Mol Biol R 2000, 64:847-867.

2. Prosser Jl, Bohannan BJM, Curtis TP, Ellis RJ, Firestone MK, Freckleton RP, Green JL, Green LE, Killham K, Lennon JJ, Osborn AM, Solan M, van der Gast $\mathrm{CJ}$, Young JPW: The role of ecological theory in microbial ecology. Nat Rev Microbiol 2007, 5:384-392.

3. Hibbing M, Fuqua C: Bacterial competition: surviving and thriving in the microbial jungle. Nat Rev Microbiol 2009, 8:15-25. 
4. Bader J, Mast-Gerlach E, Popović MK, Bajpai R, Stahl U: Relevance of microbial coculture fermentations in biotechnology. J Appl Microbio/ 2010, 109:371-387.

5. Shong J: Jimenez Diaz MR, Collins $\mathrm{CH}$ : Towards synthetic microbial consortia for bioprocessing. Curr Opin Biotech 2012, 2:1-5.

6. Brenner K, You L, Arnold FH: Engineering microbial consortia: a new frontier in synthetic biology. Trends Biotechnol 2008, 26:483-489.

7. Zuroff TR, Curtis WR: Developing symbiotic consortia for lignocellulosic biofuel production. App Microbiol Biot 2012, 93:1423-1435.

8. U.S. Deparmtent of Energy Strategic Plan DOE/CF-0067. 2011.

9. Annual Energy Review DOE/EIA-0384(2011). 2011

10. Lynd LR, Laser MS, Bransby D, Dale BE, Davison B, Hamilton R, Himmel M, Keller M, McMillan JD, Sheehan J, Wyman CE: How biotech can transform biofuels. Nat Biotechnol 2008, 26:169-172.

11. Lynd LR, Weimer PJ, ZyI WHV, Pretorius IS: Microbial cellulose utilization: fundamentals and biotechnology. Microbiol Mol Biol R 2002, 66:506-577.

12. Lynd LR, van Zyl WH, McBride JE, Laser M: Consolidated bioprocessing of cellulosic biomass: an update. Curr Opin Biotech 2005, 16:577-583.

13. Olson DG, McBride JE, Shaw JA, Lynd LR: Recent progress in consolidated bioprocessing. Curr Opin Biotech 2011, 23:396-406.

14. Ohkuma M: Termite symbiotic systems: efficient bio-recycling of lignocellulose. Int Microbio/ 2003, 61:1-9.

15. Kudo T: Termite-microbe symbiotic system and its efficient degradation of lignocellulose. Biosci Biotech Bioch 2009, 73:2561-2567.

16. Kerner A, Park J, Williams A, Lin XN: A programmable Escherichia coli consortium via tunable symbiosis. PLOS ONE 2012, 7:e34032.

17. Shou W, Ram S, Vilar JMG: Synthetic cooperation in engineered yeast populations. PNAS 2007, 104:1877-1882

18. Balagaddé FK, Song H, Ozaki J, Collins CH, Barnet M, Arnold FH, Quake SR, You L: A synthetic Escherichia coli predator-prey ecosystem. Mol Syst Biol 2008, 4:1-8.

19. You L, Cox RS, Weiss R, Arnold FH: Programmed population control by cell-cell communication and regulated killing. Nature 2004, 428:868-871.

20. Bayer TS, Widmaier DM, Temme K, Mirsky EA, Santi DV, Voigt CA: Synthesis of methyl halides from biomass using engineered microbes. J Am Chem Soc 2009, 131:6508-6515.

21. Kim HJ, Boedicker JQ, Choi JW, Ismagilov RF: Defined spatial structure stabilizes a synthetic multispecies bacterial community. PNAS 2008, 105:18188-18193.

22. Habets MGJL, Rozen DE, Hoekstra RF, de Visser JAGM: The effect of population structure on the adaptive radiation of microbial populations evolving in spatially structured environments. Ecol Lett 2006, 9:1041-1048.

23. Saxer G, Doebeli M, Travisano M: Spatial structure leads to ecological breakdown and loss of diversity. P R SOC B 2009, 276:2065-2070.

24. Warnick Thomas A, Methe Barbara A, Leschine Susan B: Clostridium phytofermentans sp. nov., a cellulolytic mesophile from forest soil. Int J Syst Evol Micr 2002, 52:1155-1160.

25. Freer SN: Utilization of glucose and cellobiose by Candida molischiana. Can J Microbiol 1995, 41:177-185.

26. Galazka JM, Tian C, Beeson WT, Martinez B, Glass NL, Cate JHD: Cellodextrin transport in yeast for improved biofuel production. Science 2010, 330:84-86.

27. Jin M, Balan V, Gunawan C, Dale BE: Consolidated bioprocessing (CBP) performance of Clostridium phytofermentans on AFEX-treated corn stover for ethanol production. Biotechnol Bioeng 2011, 108:1290-1297.

28. Gondé P, Blondin B, Leclerc M, Ratomahenina R, Arnaud A, Galzy P. Fermentation of cellodextrins by different yeast strains. Appl Environ Microb 1984, 48:265-269.

29. Freer SN, Skory CD: Production of $\beta$-glucosidase and diauxic usage of sugar mixtures by Candida molischiana. Can J Microbiol 1996, 42:431-436.

30. Veal D, Lynch J: Associative cellulolysis and dinitrogen fixation by cocultures of Trichoderma harzianum and Clostridium butyricum. Nature 1984, 310:695-696.

31. Kato S, Haruta S, Cui ZJ, Ishii M, Igarashi Y: Network relationships of bacteria in a stable mixed culture. Microb Ecol 2008, 56:403-411.

32. Paerl $\mathrm{H}$, Pinckney J: A mini-review of microbial consortia: their roles in aquatic production and biogeochemical cycling. Microb Ecol 1996, 31:225-247.

33. Kato S, Haruta S, Cui ZJ, Ishii M, Igarashi Y: Effective cellulose degradation by a mixed-culture system composed of a cellulolytic Clostridium and aerobic non-cellulolytic bacteria. FEMS Microbiol Ecol 2004, 51:133-142.

34. Maier U, Büchs J: Characterisation of the gas-liquid mass transfer in shaking bioreactors. Biochem Eng J 2001, 7:99-106.
35. Andreasen AA, Stier TJ: Anaerobic nutrition of Saccharomyces cerevisiae unsaturated fatty acid requirement for growth in a defined medium. Yeast 1950.

36. Kumar A, John L, Alam MM, Gupta A, Sharma G, Pillai B, Sengupta S: Homocysteine- and cysteine-mediated growth defect is not associated with induction of oxidative stress response genes in yeast. Biochem J 2006, 396:61-69.

37. Visser W, Scheffers WA: Batenburg-van der Vegte WH, van Dijken JP: Oxygen requirements of yeasts. Appl Environ Microb 1990, 56:3785-3792.

38. Park EY, Naruse K, Kato T: One-pot bioethanol production from cellulose by co-culture of Acremonium cellulolyticus and Saccharomyces cerevisiae. Biotechnol Biofuels 2012, 5:64

39. Taffs R, Aston JE, Brileya K, Jay Z, Klatt CG, Mcglynn S, Mallette N, Montross S, Gerlach R, Inskeep WP, Ward DM, Carlson RP: Microbial consortia: a syntrophic case study. Systems Biol 2009, 16:1-16.

40. Zomorrodi AR, Maranas CD: OptCom: A multi-level optimization framework for the metabolic modeling and analysis of microbial communities. PLOS Comput Biol 2012, 8:e1002363.

41. Biliouris K, Babson D, Schmidt-Dannert C: Stochastic simulations of a synthetic bacteria-yeast ecosystem. BMC Syst Biol 2012, 6:58.

42. Cavedon K, Leschine SB, Canale-Parola E: Cellulase system of a free-living, mesophilic Clostridium (strain C7). J Bacteriol 1990, 172:4222-4230.

doi:10.1186/1754-6834-6-59

Cite this article as: Zuroff et al:: Consortia-mediated bioprocessing of cellulose to ethanol with a symbiotic Clostridium phytofermentans/yeast co-culture. Biotechnology for Biofuels 2013 6:59

\section{Submit your next manuscript to BioMed Central and take full advantage of:}

- Convenient online submission

- Thorough peer review

- No space constraints or color figure charges

- Immediate publication on acceptance

- Inclusion in PubMed, CAS, Scopus and Google Scholar

- Research which is freely available for redistribution 